\title{
Experimental demonstration of subwavelength focusing of electromagnetic waves by labyrinth-based two-dimensional metamaterials
}

\author{
Irfan Bulu, Humeyra Caglayan, and Ekmel Ozbay \\ Department of Physics and the Nanotechnology Research Center, Bilkent University, Bilkent, 06800 Ankara, Turkey
}

\begin{abstract}
Received August 26, 2005; revised November 1, 2005; accepted November 1, 2005; posted November 8, 2005 (Doc. ID 64462)
We studied focusing in a two-dimensional metamaterial that was based on a labyrinth structure. We theoretically showed that the labyrinth-based metamaterial exhibits negative indices of refraction between 6 and 6.4 GHz. We experimentally studied the focusing effect by measuring electric field intensities on the output side of the metamaterial when the source was placed in front of the input side of the metamaterial. Our experimental results showed that it is in fact possible to focus the source field with half-widths as small as $\lambda / 4$ by using the labyrinth-based metamaterial. (C) 2006 Optical Society of America OCIS codes: 350.4010 .
\end{abstract}

Left-handed materials have recently attracted much attention in the scientific community. This new type of artificial material can provide simultaneous negative permittivity $(\epsilon)$ and permeability $(\mu)$ over a certain frequency range. ${ }^{1}$ In particular one fascinating consequence of simultaneous negative permittivity and negative permeability is the possibility of focusing electromagnetic waves beyond the diffraction limit. Pendry ${ }^{2}$ predicted that a slab with $\epsilon=-1, \mu=$ -1 may recover evanescent components of the field emitted from a source. In addition, because of negative refraction, such a medium focuses the propagating components of the source field. As a result, it may be possible to focus all of the Fourier components of the field emitted from a source. Pendry coined the term superlens for structures that have these properties. However, until now Pendry's prediction has been subject to some criticism. ${ }^{3}$

A common method for realizing simultaneous negative $\epsilon$ and $\mu$ is through the periodic arrangement of metallic wires and split-ring resonators. It was shown that the periodic arrangement of wires may provide negative $\epsilon$ values below a certain frequency. ${ }^{4}$ On the other hand, negative $\mu$ over a certain frequency range is provided by a periodic arrangement of split-ring resonators. ${ }^{5}$ However, it is now well understood that split-ring resonators have two rather serious problems, one of which is bianisotropy, ${ }^{6}$ and the other is electric coupling to the magnetic resonance. ${ }^{7}$ These two issues make it difficult to obtain isotropic left-handed structures by using a periodic arrangement of wires and split-ring resonators. We recently addressed both of these issues by introducing a modified split-ring resonator structure called the labyrinth structure. ${ }^{8}$ The schematics of the labyrinth structure are shown in Fig. 1(a). A medium composed of a periodic arrangement of labyrinth structures does not exhibit bianisotropy. In addition, the magnetic resonance of the labyrinth structure cannot be excited via incident electric fields.

In the present work we investigated the possibility of subwavelength focusing of electromagnetic waves by using a metamaterial based on the labyrinth structure. First, we theoretically showed that a medium composed of a periodic arrangement of wires and labyrinth structures exhibits negative indices of refraction. Then, we experimentally studied the focusing effect. Our results showed that it is possible to focus electromagnetic waves emitted from a finitesize source with a half-power width of $\lambda / 4$ by using a left-handed metamaterial based on the labyrinth structure.

The metamaterial medium that we used was composed of a two-dimensional periodic arrangement of wire stripes and labyrinth structures. The unit cell of the metamaterial structure is shown in Fig. 1(b). The wire stripes were printed on the back of standard FR4 substrates, and the labyrinths were printed on the front faces. The thickness of the metal, copper, was $0.05 \mathrm{~mm}$. The width of the wire stripes was $2.5 \mathrm{~mm}$. The lattice constant along the $x$ and $y$ directions was $8.8 \mathrm{~mm}(0.18 \lambda$, where $\lambda$ corresponds to $6.3 \mathrm{GHz}$ ). There were 68 layers along the $x$ direction and 5 layers along the $y$ direction. The width of the structure was $0.92 \lambda$. The height of the structure was 20 layers. This metamaterial medium has a lefthanded transmission band between 5.9 and $6.5 \mathrm{GHz}$ (Fig. 2 inset).

Several methods, such as procedures for retrieval from $S$ parameters, can be used for the determination of the index of refraction. ${ }^{9}$ Another rather

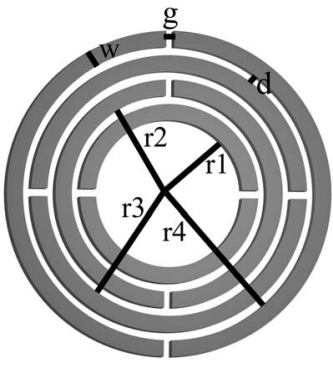

(a)

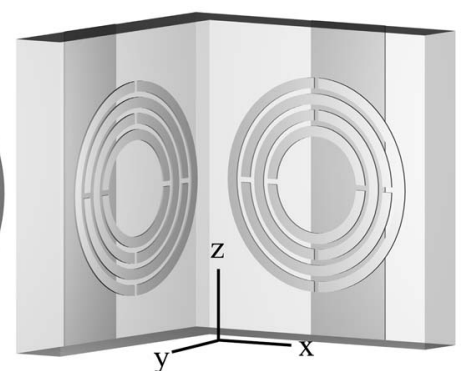

(b)
Fig. 1. (a) Labyrinth structure: $r 1=1.35 \mathrm{~mm}, r 2=1.8 \mathrm{~mm}$, $r 3=2.25 \mathrm{~mm}, r 4=2.7 \mathrm{~mm}, g=0.15 \mathrm{~mm}, w=0.3 \mathrm{~mm}$, and $d$ $=0.15 \mathrm{~mm}$. (b) Unit cell of the two-dimensional labyrinthbased left-handed metamaterial. 


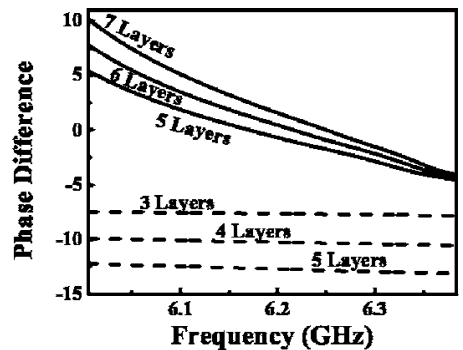

(a)

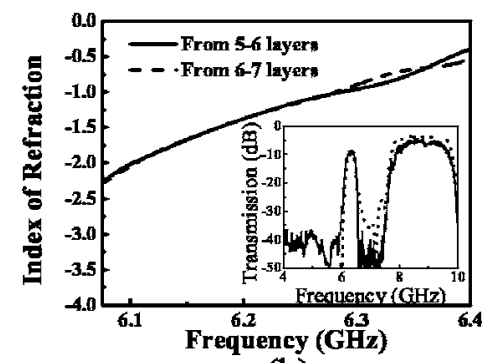

(b)

Fig. 2. (a) Phase differences between the ends of isotropic FR4 slabs (dashed curves) and the labyrinth-based metamaterial (solid curves). (b) Calculated indices of refraction for the labyrinth-based metamaterial. Inset, measured spectrum of transmission through the labyrinthbased composite metamaterial medium (solid curve) and the simulated transmission spectrum (dotted curve).

straightforward method makes use of the phase shifts when the size of the structure along the propagation direction is increased. ${ }^{10}$ It was experimentally shown ${ }^{10}$ that this method can accurately describe the real part of the index of refraction values for metamaterials even when the transmission is below $-10 \mathrm{~dB}$. Consider two pieces of homogeneous material with lengths of $L_{1}$ and $L_{2}$. The phase difference introduced due to the difference in lengths of the pieces can be written as $\Delta \phi=-\mathbf{k}_{0} n\left(L_{2}-L_{1}\right)$, where $\mathbf{k}_{0}$ is the free-space wave vector. We used the $-\mathbf{k}_{0}$ convention in this study. To theoretically determine the phase shifts when the number of layers along the propagation direction is increased, we performed finite-integration method simulations by using a commercially available software program. ${ }^{11}$ The simulation results for the phase differences between the ends of five-, six-, and seven-layer-long metamaterials are shown in Fig. 2(a). For comparison, we plotted the phase differences between the ends of three-, four-, and five-layer-long homogeneous, isotropic FR4 slabs. First, note that the phase differences for the FR4 slabs advance in the negative direction when the number of layers is increased, as is expected from a medium with a positive index of refraction, whereas the phase differences between the ends of the metamaterial advance in the positive direction with increasing length along the propagation direction. In addition, the phase differences for different lengths of metamaterials do not change significantly around $6.4 \mathrm{GHz}$. We determined the index of refraction for the metamaterial medium by using the phase shifts shown in Fig. 2(a). The results between 6.08 and $6.4 \mathrm{GHz}$ are plotted in Fig. 2(b). The frequency range was chosen in order to have a trans- mission that was above $-20 \mathrm{~dB}$ for accurate phase determination. The index of refraction is negative within this frequency range. In addition, there is a frequency range around $6.28 \mathrm{GHz}$ at which the index of refraction is close to -1 .

Encouraged by our theoretical results, we studied the focusing properties of the labyrinth-based lefthanded metamaterial. In our experiments we used monopole antennas as the source and receiver. We placed the source antenna in front of the surface of the labyrinth-based metamaterial. The axis of the source and receiver antennas were arranged parallel to the $z$ axis. The length and diameter of the monopoles were $3 \mathrm{~cm}$ and $1.5 \mathrm{~mm}$, respectively. We measured the electric field intensities over an area of $200 \times 100 \mathrm{~mm}(4.25 \lambda \times 2.1 \lambda)$ on the output side of the left-handed metamaterial in steps of $2.5 \mathrm{~mm}$ $(0.052 \lambda)$, where $\lambda$ corresponds to $6.3 \mathrm{GHz}$. The measured electric field intensities when the source antenna was placed $2 \mathrm{~cm}(0.42 \lambda)$ and $1 \mathrm{~cm}(0.21 \lambda)$ away from the input surface of the metamaterial are plotted in Figs. 3(a) and 3(b), respectively. The measurement frequency was $6.3 \mathrm{GHz}$. Note that the surface of the metamaterial was parallel to the $x$ axes. Figures 3(a) and 3(b) clearly demonstrate the focusing of the source field on the output side by the lefthanded metamaterial when the source was placed either 2 or $1 \mathrm{~cm}$ away from the input surface. The maximum field intensity along the $y$ axes was observed at $10 \mathrm{~mm}(0.21 \lambda)$ when the source was $2 \mathrm{~cm}$ $(0.42 \lambda)$ away from the input surface and at $17.5 \mathrm{~mm}$ $(0.37 \lambda)$ when the source was $1 \mathrm{~cm}(0.21 \lambda)$ away. The half-widths of the intensity profiles along the $y$ axis for both cases were $19 \mathrm{~mm}, \approx \lambda / 2.5$. Moreover, the field intensities decay as $1 / r^{2}$ along the $y$ axes, where $r$ is the distance from the focusing point. There are some extra features appearing in the image. We attribute these extra features to the interference from the waves reflected by the laboratory environment. The intensity profiles along the $x$ axis are shown in Fig. 4. In addition, we plotted the measured intensity

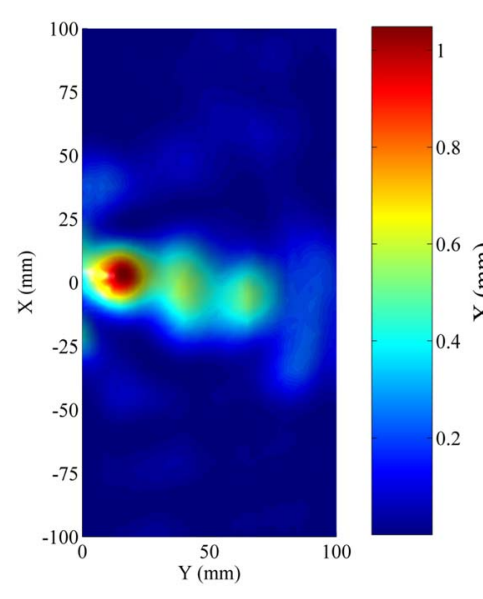

(a)

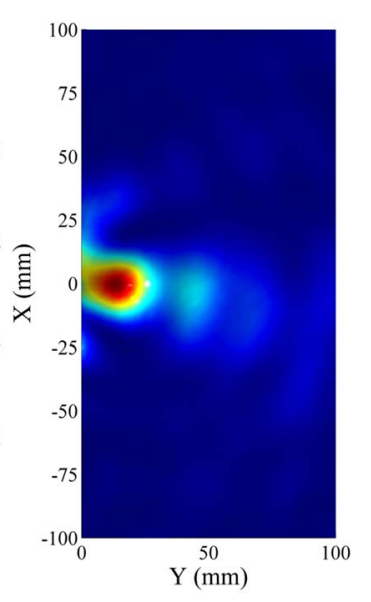

(b)
Fig. 3. (Color online) Measured electric field intensities on the output side of the metamaterial when the source was (a) 2 and (b) $1 \mathrm{~cm}$ away from the input surface of the metamaterial. 


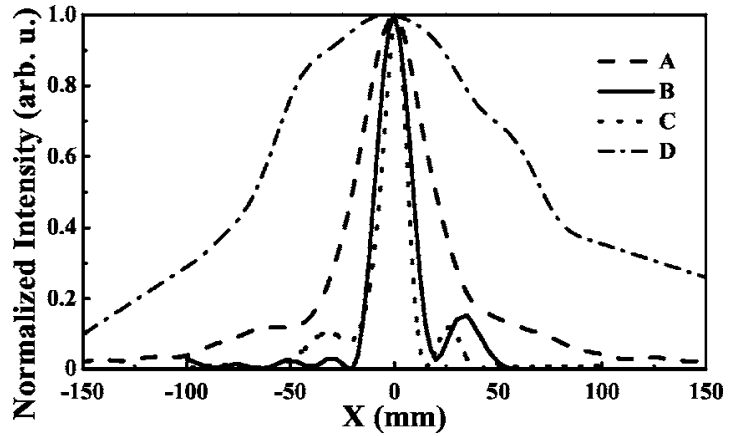

Fig. 4. Measured intensity profile of the source monopole antenna along the $x$ axis in free space when it was placed (curve A) 2 and (curve B) $8 \mathrm{~cm}$ away from the receiver antenna. Measured intensity profile along the $x$ axis on the output side of the metamaterial when the source was placed (curve C) 2 and (curve D) $1 \mathrm{~cm}$ away from the input surface of the metamaterial.

profile along the $x$ axis when the source and receiver antennas were placed $2 \mathrm{~cm}$ apart from each other in free space (dashed curve in Fig. 4). The measured half-width of the intensity profile in free space, without the metamaterial between, was $40 \mathrm{~mm}, \approx \lambda$. Without the metamaterial, even at such a close distance, the source field could not be resolved with a resolution below the wavelength. On the other hand, the half-width of the intensity profile on the output side of the metamaterial along the $x$ axes was $12 \mathrm{~mm}$, $\approx \lambda / 4$, when the source was $1 \mathrm{~cm}$ away from the input surface and was $16 \mathrm{~mm}, \approx \lambda / 3$, when the source was $2 \mathrm{~cm}$ away from the input surface. As a result it was possible to resolve the source field with a resolution below the wavelength when the metamaterial was inserted between the source and the receiver antennas. The focusing property of the metamaterial was not restricted to $6.3 \mathrm{GHz}$. We observed the focusing effect for a range of frequencies between 6.2 and $6.37 \mathrm{GHz}$, although the focusing was not as sharp as for 6.3 GHz. Note that the source and image distances do not add up to the width of the slab $(0.92 \lambda)$. We attribute this to the complex part of the index of refraction.

In conclusion, we theoretically showed that a metamaterial based on the labyrinth structure exhibits negative indices of refraction within a range of
6-6.4 GHz. Our experimental results showed that it was possible to focus the source field with a spot size as small as $\lambda / 4$. We attribute the subwavelength focusing to two major reasons. First, the index of refraction is negative. Second, the left-handed metamaterial retains the inhomogeneous plane-wave components of the source field. To our knowledge, this is the first experimental demonstration of the subwavelength focusing of electromagnetic waves by metamaterials in free space. Previously, subwavelength focusing was demonstrated by using a hardwired L-C circuit network. ${ }^{12}$ We believe that our study may find important applications in imaging. More important, our experimental results proved that metamaterials based on the labyrinth structure can focus electromagnetic waves with half-widths smaller than the wavelength.

This work was supported by the European Union under the projects EU-DALHM, EU NOEMETAMORPHOSE, EU-NOE-PHOREMOST, and TUBITAK-104E090. E. Ozbay acknowledges partial support from the Turkish Academy of Sciences. I. Bulu's e-mail address is irfan@fen.bilkent.edu.tr.

\section{References}

1. R. A. Shelby, D. R. Smith, and S. Schultz, Science 292, 77 (2001).

2. J. B. Pendry, Phys. Rev. Lett. 85, 3966 (2000).

3. M. Nieto-Vesperinas, J. Opt. Soc. Am. A 21, 491 (2004).

4. J. Pendry, A. Holden, W. Stewart, and I. Youngs, Phys. Rev. Lett. 76, 4773 (1996).

5. J. B. Pendry, A. J. Holden, D. J. Robins, and W. J. Stewart, IEEE Trans. Microwave Theory Tech. 47, 2075 (1999).

6. R. Marques, F. Medina, and R. Rafii-El-Idrissi, Phys. Rev. B 65, 144440 (2002).

7. N. Katsarakis, T. Koschny, M. Kafesaki, E. N. Economou, and C. M. Soukoulis, Appl. Phys. Lett. 84, 2943 (2004).

8. I. Bulu, H. Caglayan, and E. Ozbay, Opt. Express 13, 10238 (2005).

9. D. R. Smith, S. Schultz, P. Marko, and C. M. Soukoulis, Phys. Rev. B 65, 195104 (2002).

10. K. Aydin, K. Guven, C. M. Soukoulis, and E. Ozbay, Appl. Phys. Lett. 86, 124102 (2005).

11. User Manual Version 5.0 (CST GmbH, 2005).

12. A. Grbic and G. V. Eleftheriades, Appl. Phys. Lett. 82, 1815 (2003). 